Document downloaded from:

http://hdl.handle.net/10251/171136

This paper must be cited as:

Seimetz, M.; Bellido, P.; Soriano, A.; Huertas, C.; García Lopez, J.; Jimenez-Ramos, MC.; Fernandez, B.... (2013). Time-of-Flight Detector for the Characterisation of LaserAccelerated Protons. IEEE. 25-28. https://doi.org/10.1109/NSSMIC.2013.6829804

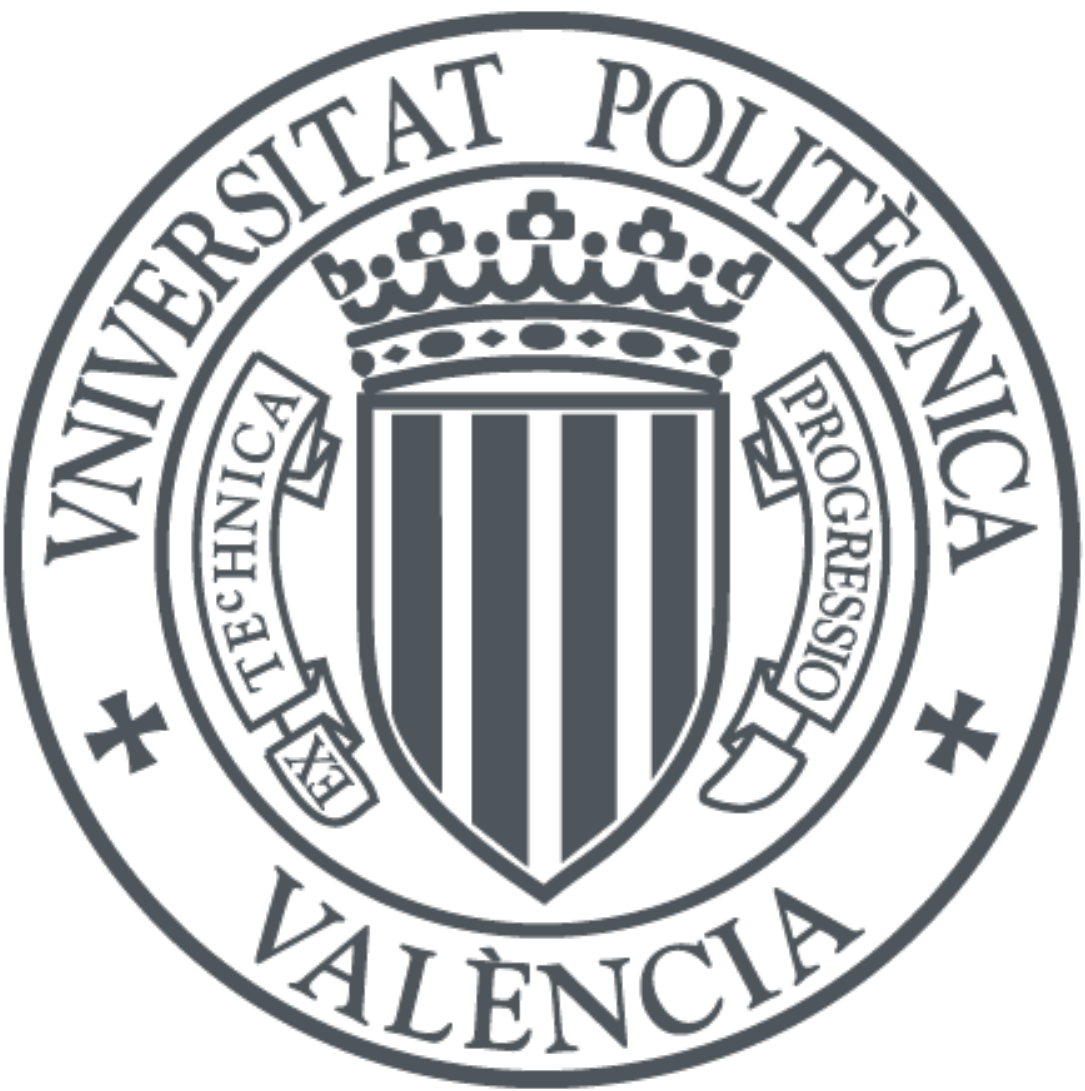

The final publication is available at

https://doi.org/10.1109/NSSMIC.2013.6829804

Copyright IEEE

Additional Information 


\title{
Time-of-Flight Detector for the Characterisation of Laser-Accelerated Protons
}

\author{
M. Seimetz*, P. Bellido, A. Soriano, C. Huertas, J. García López, M. C. Jiménez-Ramos, B. Fernández, P. Conde, \\ E. Crespo, A. J. González, L. Hernández, A. Iborra, F. Martos, L. Moliner, J. P. Rigla, M. J. Rodríguez-Álvarez, \\ F. Sánchez, L. F. Vidal, and J. M. Benlloch
}

\begin{abstract}
Lasers of ultra-high intensity focused on thin targets can form plasmas and release large numbers of charged particles with kinetic energies in the $\mathrm{MeV}$ region. The characterization of the accelerated particles requires suitable detectors. We present a time-of-flight detector based on a plastic scintillator optimized for the spectral analysis of laser-accelerated protons.

All details of the detector layout are adapted to the expected properties of the proton beam. Particle energies will be separated by the time-of-flight technique over $200 \mathrm{~cm}$ path length. The active area $(25 \mathrm{~mm}$ width) corresponds to a few mrad opening angle. With $5 \mathbf{~ m m}$ thickness the detector is capable of absorbing protons up to $22.5 \mathrm{MeV}$. A very thin, aluminized mylar foil shields the scintillator from outer light while absorbing very little particle energy. The scintillation photons are measured with a photomultiplier tube coupled through a bundle of optical fibres. The coupling of these fibres via a PMMA light guide has been previously optimized in simulations with Litrani.

A critical aspect of the detection of virtually large numbers of protons emitted in femtosecond pulses is the saturation of the PMT. The latter can be avoided by use of appropriate optical filters. With these the effective dynamic range starts from single particles over several orders of magnitude.

Our time-of-flight detector has been calibrated at the Spanish National Accelerator Centre at Sevilla. Proton beams from 0.46 to 5.6 MeV from a tandem accelerator have been used to measure the relation between particle energy and pulse heights. Further tests have been performed with a pulsed electron beam to simulate many-particle hits.
\end{abstract}

Index Terms-Laser ion acceleration, time-of-flight measurement, proton detector

\section{INTRODUCTION}

$\mathbf{P}$ ROTON and ion acceleration by ultra-short, ultra-intense laser pulses has become a very active research field throughout the last decade [1]. Several high-power laser facilities have demonstrated the production of proton pulses over

Manuscript received November 11, 2013. Project funded by the Spanish Ministry of Economy and Competitiveness and co-funded with FEDER's funds within the INNPACTO 2011 program, Grant No. IPT-2011-0862900000. This work was supported by the Spanish Plan Nacional de Investigación Científica, Desarrollo e Innovación Tecnológica (I+D+i) under Grant No. FIS2010-21216-CO2-01 and the Valencian Local Government under Grants PROMETEOII/2013/010 and ISIC 2011/013.

M. Seimetz, P. Bellido, A. Soriano, C. Huertas, P. Conde, E. Crespo, A. J. González, L. Hernández, A. Iborra, F. Martos, L. Moliner, J. P. Rigla, M. J. Rodríguez-Álvarez, F. Sánchez, L. F. Vidal, and J. M. Benlloch are with Instituto de Instrumentación para Imagen Molecular (I3M), CSIC Universidad Politécnica de Valencia - CIEMAT, Camino de Vera s/n, Ed. 8-N-1, 46022 Valencia, Spain.

J. García López, M. C. Jiménez-Ramos, and B. Fernández are with Centro Nacional de Aceleradores (CNA), U. Sevilla - J. Andalucía - CSIC, Avda. Thomas Alva Edison 7, 41092 Sevilla, Spain.

*Corresponding author. Contact: mseimetz@i3m.upv.es. a broad energy range. The number and energy spectrum of the accelerated particles vary strongly with laser parameters such as the pulse power, focused intensity, prepulse duration, and contrast, but also with the target material and thickness. A deeper understanding of the underlying mechanisms in different intensity regimes and the optimisation of experimental conditions are key aspects of current research activities. They require systematic studies comprising the spectral analysis of the accelerated protons and ions.

The Institute for Instrumentation in Molecular Imaging (I3M, Valencia) is collaborating in a new laser-plasma experiment located at the Spanish Pulsed Laser Centre (CLPU), at Salamanca. For the first data taking period a time-offlight (TOF) detector has been designed and developed for the characterisation of $\mathrm{MeV}$-energy protons in bunches with large numbers of particles. Similar TOF detectors have been applied by other laser-plasma collaborations [2], [3]. The design requirements, deduced from expected proton spectra, will be summarised in section II. The setup of our detector and the optimisation of its components will be presented in section III. Finally, a series of tests demonstrating the functionality will be detailed in section IV.

\section{DESIGN REQUIREMENTS}

In our initial measurements at CLPU-Salamanca we will work at focused laser intensities of the order $10^{18}-10^{19} \mathrm{~W} / \mathrm{cm}^{2}$ and around $1 \mathrm{~J}$ pulse energy. Other experiments under similar conditions observed protons with $1-10 \mathrm{MeV}$ maximum energy [1]. Our detector should be able to cover at least the same range. The corresponding momenta can be resolved by their time-of-flight difference over $150-200 \mathrm{~cm}$ path length behind the laser target. We expect to observe overlapping pulses of a large number of particles between 50 and $200 \mathrm{~ns}$ after the laser shot. The single-particle timing resolution should be much better (a few nanoseconds).

The biggest uncertainty resides in the number of detected protons. Published results vary between a few hundred and $\sim 10^{12}$ protons per laser shot, mainly depending on the target material, thickness, and structure. As a consequence, our aim is to design a versatile detector with a very large dynamic range. Furthermore, for repeated laser shots we require realtime capability (unlike, for example, radiochromic films). A strong electromagnetic pulse emitted by the plasma may disturb the readout or even damage electronic devices. These should therefore be enclosed in a shielded environment at some distance from the detector. 


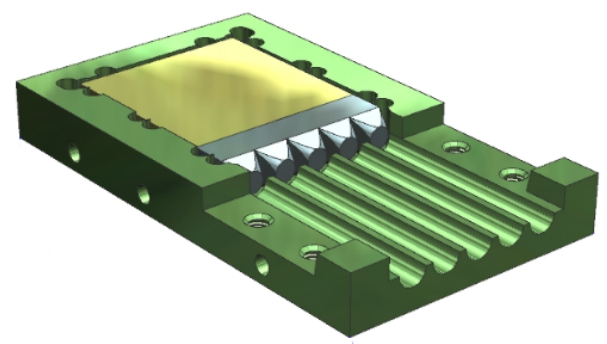

Fig. 1. Plastic scintillator and light guide in their protective housing.

All these requirements are met by a plastic scintillator coupled to a photomultiplier tube (PMT) via a bundle of optical fibres as described in the following section. Silicon photomultipliers (SiPMs) may be an interesting alternative to the classical PMT, however we have chosen the latter for its extensive use and wide dynamic range. Further, it allows for simple merging of the fibres on a common photocathode. Its analogue output pulses are recorded on an oscilloscope with $1 \mathrm{GHz}$ sampling rate (Agilent Technologies MSO7104B). This allows for the characterisation and calibration of single-particle pulses as well as the measurement of the height and shape of broad, overlapping signals from a large number of protons within bunches of $200 \mathrm{~ns}$ length.

\section{SETUP AND OPTIMISATION}

The sensitive part of the time-of-flight detector is made of a plastic scintillator with $25 \times 25 \mathrm{~mm}^{2}$ active area. It provides about $10^{3}$ scintillation photons per proton (at $1 \mathrm{MeV}$ ) and quick decay time ( $2 \mathrm{~ns}$ ). Its $5 \mathrm{~mm}$ thickness is sufficient to stop protons up to $22.5 \mathrm{MeV}$. Thus, a unique relation between measured pulse heights and particle energies is guaranteed. The scintillator is wrapped with two layers of $2 \mu \mathrm{m}$ thick aluminised mylar for light protection at minimum energy loss. It is mounted in a protective housing which guarantees a mechanically stable connection of the optical fibres (Fig. 1).

The scintillator is coupled to the PMT via a bundle of 5 optical fibres $\left(E^{2 k a^{\mathrm{TM}}} \mathrm{CK}-120,3 \mathrm{~mm} \varnothing\right)$. In initial tests these relatively thick fibres have shown a significantly better transmission efficiency than bundles of thinner fibres $(0.75 \mathrm{~mm})$, but the same total diameter. Thus, a large part of the output area is covered, with small dead space between the fibres. A drawback of this choice is the mechanical rigidity, however for our purpose the thick fibres are sufficiently flexible.

The coupling between the scintillator and the fibres has been optimized in the following way. First, each circular fibre corresponds to a $5 \times 5 \mathrm{~mm}^{2}$ square part of the side face of our scintillator. A tapered light guide made of PMMA is placed in between to avoid dead spaces outside the fibre area. Geometrical considerations show that the inclination angle of this truncated cone should correspond to the angle of internal reflection of the fibre.

Second, we have simulated the uniformity of the scintillator's response using the SLitrani program [4]. This software allows for modelling the production and propagation of scintillation light in optical media, taking into account the detailed properties of couplings and external materials. We

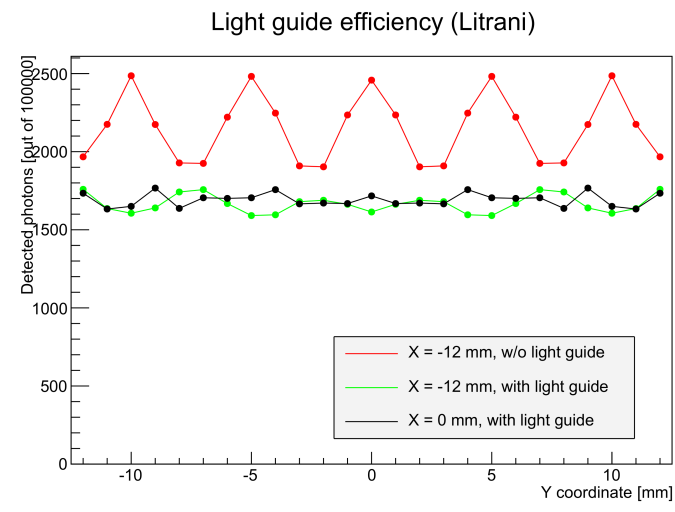

Fig. 2. Results of simulations of the light collection efficiency.

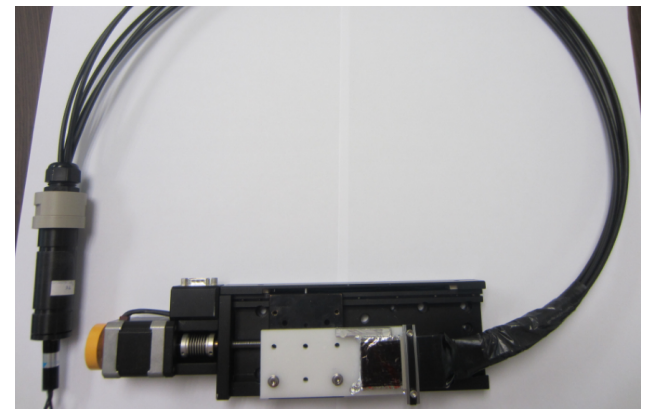

Fig. 3. Our scintillator (here mounted on a support) coupled to the PMT.

have simulated the total number of detected photons on a PMT (at the opposite end of the fibres) as a function of the position of a uniform light source inside the scintillator (corresponding to a series of proton hit positions). This source has been moved along the $Y$ coordinate, parallel to the scintillator output face. With the tapered light guides alone we have found large, position-dependent deviations of the number of detected photons, amounting up to $20 \%$ when the source is close to the output face $(X=-12 \mathrm{~mm})$, see Fig. 2. If we place an additional, rectangular piece made of PMMA between the scintillator and the cones these differences virtually disappear. Further, along a line through the centre of the scintillator $(X=0 \mathrm{~mm})$ we obtain almost the same number of photons. We thus conclude that this configuration, which can be seen in Fig. 1, provides much better uniformity of proton detection. The absolute light transmission efficiency according to the simulations is around $8 \%$ for scintillation light emitted uniformly from random interaction points inside the active volume.

The scintillation light is converted into electronic pulses with a Hamamatsu R647 photomultiplier tube $(10 \mathrm{~mm}$ active diameter). All five optical fibres are dry-coupled to its entrance window. Variable optical filters between the fibres and PMT allow for adjustment of the dynamic range. In our initial tests we have applied neutral density filters with OD1-3 although higher optical densities may be necessary to avoid PMT saturation at large numbers of particles.

The complete detector assembly is shown in Fig. 3, with the scintillator mounted on a support and the PMT inside a protective housing. 

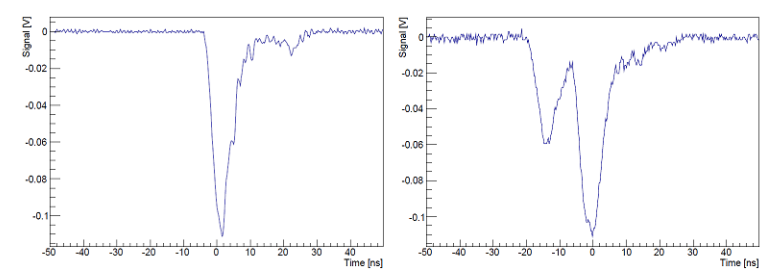

Fig. 4. Oscilloscope pulse of a $\gamma$ source (left). Right: Pulses originating from a single proton followed by two protons.

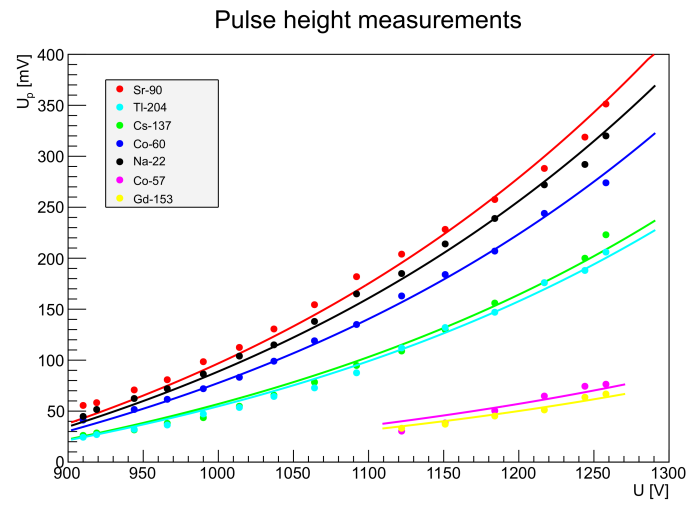

Fig. 5. Maximum oscilloscope pulse heights of $\gamma$ and $\beta$ sources over a range of photomultiplier voltages.

\section{TEST PROCEDURES AND RESULTS}

We have tested the performance of the scintillator in a series of measurements. First, we have explored its response to different $\gamma$ and $\beta$ sources throughout a range of energies. For each isotope we have identified the highest pulses on the oscilloscope corresponding to the $\gamma$ Compton edge and $\beta$ spectrum end point, respectively. An example from a ${ }^{22} \mathrm{Na}$ source is shown in Fig. 4 (left). Note that the highest energy emitted from this nucleus is $1275 \mathrm{keV}$ corresponding to a Compton edge of $1062 \mathrm{keV}$. The pulses show a very good signal-to-noise ratio and a width of approximately 6 ns FWHM.

We have varied the PMT high voltage between 910 and $1260 \mathrm{~V}$ resulting in a series of data points for each isotope (Fig. 5). These have been fitted with an exponential function,

$$
U_{p}=p_{0} e^{p_{1}(U-900 \mathrm{~V})+p_{2}},
$$

where the parameters $p_{1}$ and $p_{2}$ are fixed. We have found an approximately linear relation between the only free parameter, $p_{0}$, and the deposited energy.

A second test series has been performed on a $3 \mathrm{MV}$ tandem accelerator at the Spanish National Accelerator Centre (CNA, Sevilla). At the lowest possible beam currents (below $1 \mathrm{pA}$ ) pulses from single protons could be identified on the oscilloscope. Sometimes signals with approximately twice the height could be observed. These are identified as two protons arriving at the same time (Fig. 4). The beam energy has been varied over the largest possible range. We have clearly identified protons with energies as low as $0.46 \mathrm{MeV}$ up to $5.6 \mathrm{MeV}$, always at the lowest possible high voltage setting $(910 \mathrm{~V})$. The corresponding maximum pulse heights of single and double events are shown in Fig. 6. An approximately

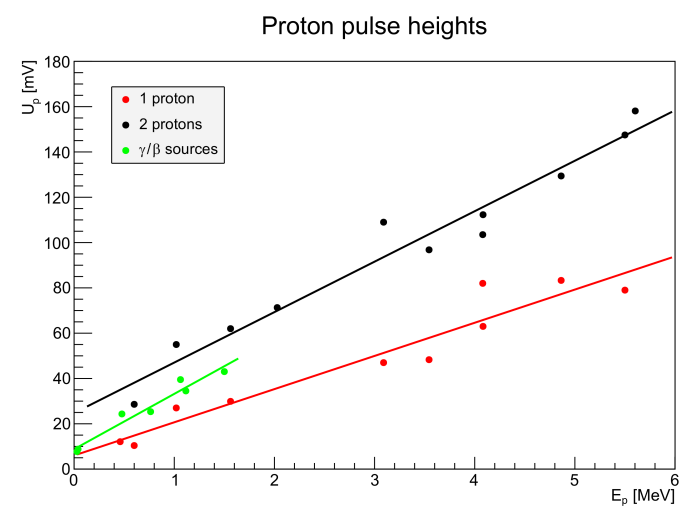

Fig. 6. Maximum oscilloscope pulse heights of single and double proton pulses over a range of energies.

linear relation between proton energy and signal height has been found. The green data points indicate the measurements for the $\gamma$ and $\beta$ sources discussed above. At a given energy a somewhat lower signal for protons can be explained by saturation effects inside the scintillator.

The clear identification of protons at $0.46 \mathrm{MeV}$ is important because it demonstrates that these particles are able to pass through the mylar film and that their scintillation light, emitted within a few microns close to the entrance surface, is sufficient to produce clearly detectable signals on the oscilloscope.

The aim of the third test series has been to study the response of our detector to large numbers of particles in long bunches. This has been done on an electron accelerator of type Siemens Primus ${ }^{\mathrm{TM}}$ which is designed for radiation therapy. It provides $3 \mu$ s long pulses with $4.5-20 \mathrm{~ms}$ separation. We have made use of the lowest possible electron energy, $6 \mathrm{MeV}$.

Our scintillator was placed about $160 \mathrm{~cm}$ below the builtin electron collimator. The irradiated field was $30 \times 30 \mathrm{~mm}^{2}$ wide, i.e. sufficient to illuminate the entire scintillator. Part of the detector was covered by tungsten plates of $6 \mathrm{~mm}$ total thickness. The aperture of this collimator was changed in various steps to study the detector response to different numbers of particles. Furthermore, independent test series were performed with different optical filters in front of the PMT.

Figure 7 shows the measured, $3 \mu$ s long pulses for different separations of the tungsten plates. They are not perfectly rectangle-shaped, but have a strong dip around $t=250 \mathrm{~ns}$. At least up to $6.2 \mathrm{~mm}$ aperture one can observe a broad signal at a constant level. This is less evident for $25 \mathrm{~mm}$ opening, i.e. irradiation of the entire scintillator surface. The latter pulse breaks down at $t \simeq 1300 \mathrm{~ns}$, a clear sign of PMT saturation. In Fig. 8 this becomes still more evident. Here the signal level is plotted as function of the tungsten aperture. With the OD1 filter an approximately linear rise is visible for small openings, but at $25 \mathrm{~mm}$ saturation is obvious. With an OD2 filter the correlation is linear throughout the entire range, albeit with very small pulse heights.

A final saturation test has been performed with a light pulser (model BNC 6010, Berkeley Nucleonics Corporation). $3.7 \mu$ s long pulses at $450 \mathrm{~nm}$ wavelength were injected directly 


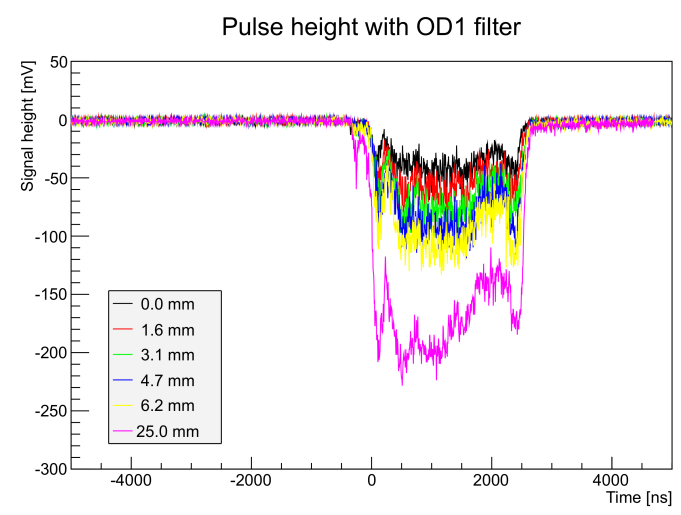

Fig. 7. Oscilloscope pulses obtained from electron bunches and different collimator apertures.

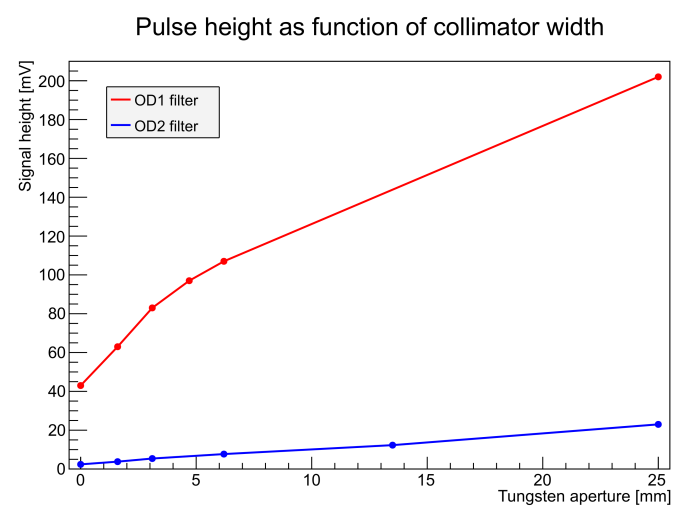

Fig. 8. Pulse heights observed as function of collimator apertures.

into the PMT (not via the scintillator and fibres). With an intermediate OD1 filter an arbitrary initial amplitude of the light pulse marked the $100 \%$ level (Fig. 9). We found that a signal level of $100 \mathrm{mV}$ at the $50 \Omega$ input resistor of the oscilloscope can be maintained throughout the entire pulse duration. This makes us confident that the dips in the electron pulses (Fig. 7) reflect true instabilities of the particle intensity and are not artefacts of the photomultiplier response. At $150 \mathrm{mV}$ initial pulse height the signal shows a negative slope losing $\sim 10 \%$ over its entire length. A $200 \mathrm{mV}$ signal rapidly breaks down. All these tests show that the PMT readout can adequately reproduce long, multi-particle pulses and that its saturation can be avoided by careful choice of intermediate optical filters.

\section{Conclusions}

We have designed an instrument based on a plastic scintillator for the detection and time-of-flight measurement of protons at energies up to $22.5 \mathrm{MeV}$. Its readout via a PMT, combined with variable optical filters, allows for adjustment over a wide range of particle numbers coinciding within a $200 \mathrm{~ns}$ long interval. Thus the particle spectrum can be reconstructed from the overlap pulse. The coupling between scintillator and PMT based on optical fibres has been optimised in previous simulations, including the design of an intermediate light guide.

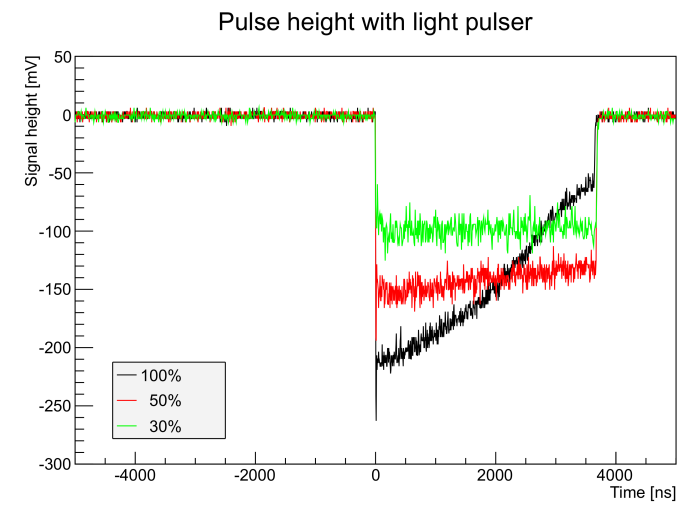

Fig. 9. PMT output pulses from light generator.

The detector has been tested with several $\gamma$ and $\beta$ sources. We have demonstrated its capability to detect protons with energies starting from $0.46 \mathrm{MeV}$ as well as many-particle pulses.

The scintillator is to be applied for the detection of laseraccelerated protons at the Spanish Pulsed Laser Centre in the near future.

\section{ACKNOWLEDGMENTS}

The authors wish to thank Aurora Vicedo from Hospital General (Valencia) for the opportunity and support to realise detector tests with a therapeutic electron accelerator.

\section{REFERENCES}

[1] H. Daido et al., "Review of laser-driven ion sources and their applications", Rep. Prog. Phys. 75, 056401 (2012)

[2] A. Yogo et al., "Laser prepulse dependency of proton-energy distributions in ultraintense laser-foil interactions with an online time-of-flight technique", Phys. Plasmas 14, 043104 (2007).

[3] I. W. Choi et al., "Ion spectrometer composed of time-of-flight and Thomson parabola spectrometers for simultaneous characterization of laser-driven ions", Rev. Sci. Instrum. 80, 053302 (2009).

[4] F. X. Gentit, "Litrani: A General purpose Monte Carlo program simulating light propagation in isotropic or anisotropic media", Nucl. Inst. Meth. 486, 35-39 (2002); http://gentitfx.fr/litrani/. 